\title{
Non-alcoholic fatty liver disease in cerebrovascular disease: a bystander or a real risk factor?
}

\author{
Lung-Yi Mak $^{1,2} \cdot$ James Fung ${ }^{2,3}$ (1)
}

Received: 21 September 2021 / Accepted: 7 November 2021 / Published online: 27 January 2022

(c) Asian Pacific Association for the Study of the Liver 2022

Over the past decade, non-alcoholic fatty liver disease (NAFLD) has emerged to become one of the most common causes of decompensated cirrhosis, and the leading primary liver disease etiology for liver transplantation. Although NAFLD is a well-established risk factor for cardiovascular disease, its association with cerebrovascular disease is less conclusive. Furthermore, the risk factors that are associated with the development of NAFLD, including obesity, diabetes mellitus, hyperlipidemia, and hypertension, are similar to those that increase the risk of cerebrovascular events. Although the association between NAFLD and cerebrovascular events has been reported, whether NAFLD is a true independent risk factor for strokes or a mere consequence of the shared metabolic risk factors remains to be determined.

In a meta-analysis of nine studies, NAFLD increased the risk of strokes $(\mathrm{OR}=2.32,95 \% \mathrm{CI} 1.84-2.93, p<0.001)$, and the effect was observed in both Caucasians and Asians [1]. However, in a recent study of over 120,000 adults with a diagnosis of NAFLD/NASH, after adjusting for cardiovascular risk factors, NAFLD was not found to be associated with an increased stroke risk [2]. There may be many factors accounting for the discrepancies in results in these cohort studies [3-7]. Selection bias is unavoidable, and those NAFLD patients included in specialist referral centres may have more risk variables than those in the primary care settings, and may undergo significant lifestyle changes after being diagnosed. Important data may not be captured, such as incidental NAFLD cases during follow-up for control

\section{James Fung}

jfung@gastro.hk

1 Department of Medicine, Queen Mary Hospital, The University of Hong Kong, Hong Kong, China

2 State Key Laboratory of Liver Research, The University of Hong Kong, Hong Kong, China

3 Department of Medicine, Queen Mary Hospital, The University of Hong Kong, Room 305, New Clinical Building, Pokfulam Road 102, Hong Kong, China groups, medication usage including anti-diabetic and antilipid drugs, and physical activity. The longer duration of follow-up that is needed to assess stroke endpoints may lead to large numbers being lost to follow-up, low number of patients with endpoints, with increase in type 2 error. The different modalities used to diagnose NAFLD between studies will also limit the comparability between these studies. Therefore, the extent to which NAFLD increases the risk of stroke remains debatable. Furthermore, it is possible that non-alcoholic steatohepatitis (NASH) rather than bland steatosis, and the presence of liver fibrosis, both of which are not detected on imaging, increases the risk of stroke [8].

The mechanistic effect whereby NAFLD increases the risk of stroke remains unclear, although current evidence suggests that it is underpinned by a chronic inflammatory state. This may create a prothrombotic tendency with increase in platelet activity and endothelial dysfunction. Upregulation of pro-atherogenic transcription factors may also lead to the development of atherosclerotic lesions. Indeed, higher carotid arterial stiffness and increase in arterial wall thickness and thickness of the intima-media index of the internal carotid artery has been associated with NAFLD, which may increase the risk of subsequent stroke events [9, 10]. Furthermore, vascular damage may occur with oxidative stress due to the production of reactive oxygen species as a result of mitochondrial dysfunction. Apart from increase in platelet activity, NAFLD may also be associated with increase in tissue plasminogen activator-1 inhibitor and factor VIII, IX, XI, and XII activities, and a reduction in Protein $\mathrm{C}$, thereby further increasing the prothrombotic imbalance $[11,12]$. Finally, NAFLD may also contribute to the unfavourable lipid profile that may heighten the risk of stroke and self-perpetuate the hepatic fat deposition. NAFLD has been associated with the atherogenic dyslipidemia phenotype, secondary to the overproduction of larger triglyceriderich very low-density lipoprotein (VLDL) particles from the liver [13]. The effects of increase lipid synthesis together 
with underlying insulin resistance may result in a higher triglyceride and lower HDL-cholesterol level.

The retrospective cross-sectional study conducted by Mori et al. in the current issue evaluated the frequency of NAFLD and the risk factors associated with the development of NAFLD in 676 acute stroke patients aged 40-79 years. Using ultrasound, 267 (39.5\%) of patients had NAFLD, which was comparatively higher than the previously reported $26.4 \%$ from the general Japanese population. In general, the prevalence of NAFLD in patients experiencing cerebrovascular events is quite variable, ranging from 8 to $44 \%$ [14]. When compared to those without NAFLD in the current study, patients with NAFLD were younger, had higher levels of liver enzymes, serum albumin, HbA1C, serum lipids (excluding LDL-cholesterol), blood pressure, and anthropometric values, and lower AST/ALT ratio. In addition, higher levels of lauric acid, myristic acid, palmitic acid (PA), stearic acid, oleic acid, linoleic acid, dihomo-gamma-linolenic acid (DGLA), arachidonic acid (AA), alpha-linolenic acid, and docosahexaenoic acid (DHA) were observed in patients with NAFLD, whereas no difference was found in eicosapentanoic acid.

After multivariate analysis, high BMI, low AST/ALT ratio, high albumin, PA\% and DGLA levels were independently associated with NAFLD in acute stroke patients. In contrast, other well-known risk factors including blood pressure, glucose level, HbA1C level, total cholesterol and triglycerides were not independent factors. A high BMI is not surprising and is a well-established risk factor. Similarly, there is data showing a positive correlation between ALT/ AST ratio with NAFLD risk and hepatic fat infiltration. A higher ALT may indicate underlying inflammatory activity, and chronic inflammation may induce insulin resistance and steatosis in the liver. The finding of a high albumin as an independent risk factor is unexpected, and somewhat paradoxical, as a lower albumin would be anticipated, especially for those with advanced liver disease. In the early stages of liver disease, it would be unusual for any significant differences between NAFLD and non-NAFLD patients. Unfortunately, the authors do not offer any potential explanations for this finding. Whether the association of higher albumin with NAFLD is peculiar to acute stroke patients remains to be determined. It is also possible that non-NAFLD patients may have underlying renal dysfunction or other chronic inflammatory disease that was not captured. Finally, this may be due to the limitations of the statistics employed using only variables without multicollinearity as defined by a low variance inflation factor of less than 3 .

Despite this, the current study may shed light on the role of different fatty acids on NAFLD in acute stroke patients. The same group has published on the association of serum fatty acids and higher serum DGLA levels with the age of onset of acute ischemic stroke and intracerebral haemorrhage
$[15,16]$. DGLA is commonly used as a health supplement for its alleged effects in a variety of diseases, including suppression of chronic inflammation, vasodilation and blood pressure lowering, and inhibition of progression of atherosclerosis [17]. On the other hand, DGLA is metabolized to arachidonic acid, and subsequently into prostaglandin E2, thromboxane $\mathrm{A} 2$, or leukotriene $\mathrm{C} 4$, thereby potentially increasing the risk of cerebrovascular events by the virtue of a pro-inflammatory state. Therefore, it would appear that the types of fatty acids and their metabolites may determine the quality of the triglyceride, and some in fact may not be detrimental. A previous study has shown that individual serum trans, saturated, and monosaturated fatty acids were positively associated with ischemic stroke, in contrast to individual $\mathrm{n} 3$ and $\mathrm{n} 6$ polyunsaturated fatty acids where there was an inverse relationship [18]. It would be important to identify why some patients have higher DGLA than the others, and a detailed dietary history would be helpful.

Before we can draw any firm conclusions from the study, there are several aspects to consider. First, the issue with higher serum albumin remains unresolved. Second, as with all cross-sectional studies, reverse causality remains a possibility, and selection bias cannot be eliminated. Thirdly, the diagnosis of NAFLD was based on ultrasound only. Last, and most importantly, a comparison arm consisting of nonstroke patients is lacking, therefore, we cannot ascertain whether the current findings are specific for acute stroke patients, and why one would expect the risk factors to vary in stroke, as compared to other cardiovascular diseases. Furthermore, the lack of a control group means that we cannot determine whether there is any actual association of NAFLD and stroke. Ultimately, acute stroke is the more important endpoint, and a control arm is required to determine if factors associated with NAFLD development are also important in stroke occurrence. Identifying risk factors associated with NAFLD in acute stroke patients in itself has less clinical relevance, especially in the absence of liver sequelae.

There is evidence to suggest that the presence of NAFLD not only can increase the risk of stroke, but also impact on the outcome after a stroke event, although the results have been contradictory. Some studies have shown that NAFLD may be associated with more severe stroke and worse outcome [19]. Paradoxically, another study showed that a higher burden of hepatic steatosis was associated with less severe stroke and better functional outcome [20]. It remains possible that the underlying liver fibrosis and not steatosis is responsible for outcomes after stroke [21]. Indeed, the presence of liver fibrosis has been shown to be associated with unfavourable long-term prognosis in ischemic stroke or transient ischemic attack patients [8, 21].

As the relationships between NAFLD, steatosis, fibrosis, stroke risk, and stroke outcome are complex, well-designed randomized controlled trials with adequate follow-up and 
sample size to demonstrate a reduction in incidence of stroke with treatment of NAFLD may be a valuable addition in filling the lacunae in evidence so far. A standardized method for the diagnosis of NAFLD should be adopted and repeated at intervals along with updated histories on medication, physical activity, dietary modification, and the development of metabolic disorders. This will allow identification of risk factors underlying NAFLD and cerebrovascular disease, assessment of their dynamic impact on stroke development, and allowing better management directed at reducing these risks.

\section{Declarations}

Conflict of interest There is no conflict of interest.

\section{References}

1. Hu J, Xu Y, He Z, Zhang H, Lian X, Zhu T, et al. Increased risk of cerebrovascular accident related to non-alcoholic fatty liver disease: a meta-analysis. Oncotarget 2018;9:2752-2760

2. Alexander M, Loomis AK, van der Lei J, Duarte-Salles T, PrietoAlhambra D, Ansell D, et al. Non-alcoholic fatty liver disease and risk of incident acute myocardial infarction and stroke: findings from matched cohort study of 18 million European adults. BMJ 2019;367:15367

3. Hamaguchi M, Kojima T, Takeda N, Nagata C, Takeda J, Sarui $\mathrm{H}$, et al. Nonalcoholic fatty liver disease is a novel predictor of cardiovascular disease. World J Gastroenterol 2007;13:1579-1584

4. Domanski JP, Park SJ, Harrison SA. Cardiovascular disease and nonalcoholic fatty liver disease: does histologic severity matter? J Clin Gastroenterol 2012;46:427-430

5. Pickhardt PJ, Hahn L, MunozdelRio A, Park SH, Reeder SB, Said A. Natural history of hepatic steatosis: observed outcomes for subsequent liver and cardiovascular complications. AJR Am J Roentgenol 2014;202:752-758

6. El Azeem HA, el Khalek SA, El-Akabawy H, Naeim H, Khalik HA, Alfifi AA. Association between nonalcoholic fatty liver disease and the incidence of cardiovascular and renal events. J Saudi Heart Assoc 2013;25:239-246

7. Fracanzani AL, Tiraboschi S, Pisano G, Consonni D, Baragetti A, Bertelli C, et al. Progression of carotid vascular damage and cardiovascular events in non-alcoholic fatty liver disease patients compared to the general population during 10 years of follow-up. Atherosclerosis 2016;246:208-213
8. Baik M, Kim SU, Kang S, Park HJ, Nam HS, Heo JH, et al. Liver fibrosis, not steatosis, associates with long-term outcomes in ischaemic stroke patients. Cerebrovasc Dis 2019;47:32-39

9. Lee YJ, Shim JY, Moon BS, Shin YH, Jung DH, Lee JH, et al. The relationship between arterial stiffness and nonalcoholic fatty liver disease. Dig Dis Sci 2012;57:196-203

10. Jaruvongvanich V, Chenbhanich J, Sanguankeo A, Rattanawong $\mathrm{P}$, Wijarnpreecha K, Upala S. Increased arterial stiffness in nonalcoholic fatty liver disease: a systematic review and meta-analysis. Eur J Gastroenterol Hepatol 2017;29:e28-e35

11. Tripodi A, Fracanzani AL, Primignani M, Chantarangkul V, Clerici M, Mannucci PM, et al. Procoagulant imbalance in patients with non-alcoholic fatty liver disease. J Hepatol 2014;61:148-154

12. Kotronen A, Joutsi-Korhonen L, Sevastianova K, Bergholm R, Hakkarainen A, Pietilainen KH, et al. Increased coagulation factor VIII, IX, XI and XII activities in non-alcoholic fatty liver disease. Liver Int 2011;31:176-183

13. DeFilippis AP, Blaha MJ, Martin SS, Reed RM, Jones SR, Nasir $\mathrm{K}$, et al. Nonalcoholic fatty liver disease and serum lipoproteins: the multi-ethnic study of atherosclerosis. Atherosclerosis 2013;227:429-436

14. Lombardi R, Fargion S, Fracanzani AL. Brain involvement in non-alcoholic fatty liver disease (NAFLD): a systematic review. Dig Liver Dis 2019;51:1214-1222

15. Mori T, Yoshioka K, Tanno Y, Kasakura S. Association of serum fatty acids at admission with the age of onset of intracerebral hemorrhage. Nutrients 2020;12:31

16. Mori T, Yoshioka K, Tanno Y, Kasakura S. Association of serum fatty acids at admission with the age of onset of acute ischemic stroke. Nutrients 2020;12:65

17. Fan YY, Chapkin RS. Importance of dietary gamma-linolenic acid in human health and nutrition. J Nutr 1998;128:1411-1414

18. Yaemsiri S, Sen S, Tinker LF, Robinson WR, Evans RW, Rosamond W, et al. Serum fatty acids and incidence of ischemic stroke among postmenopausal women. Stroke 2013;44:2710-2717

19. Li H, Hu B, Wei L, Zhou L, Zhang L, Lin Y, et al. Non-alcoholic fatty liver disease is associated with stroke severity and progression of brainstem infarctions. Eur J Neurol 2018;25:577-e534

20. Baik M, Kim SU, Nam HS, Heo JH, Kim YD. The paradoxical protective effect of liver steatosis on severity and functional outcome of ischemic stroke. Front Neurol 2019;10:375

21. Baik M, Nam HS, Heo JH, Park HJ, Kim BK, Park JY, et al. Advanced liver fibrosis predicts unfavorable long-term prognosis in first-ever ischemic stroke or transient ischemic attack. Cerebrovasc Dis 2020;49:474-480

Publisher's Note Springer Nature remains neutral with regard to jurisdictional claims in published maps and institutional affiliations. 